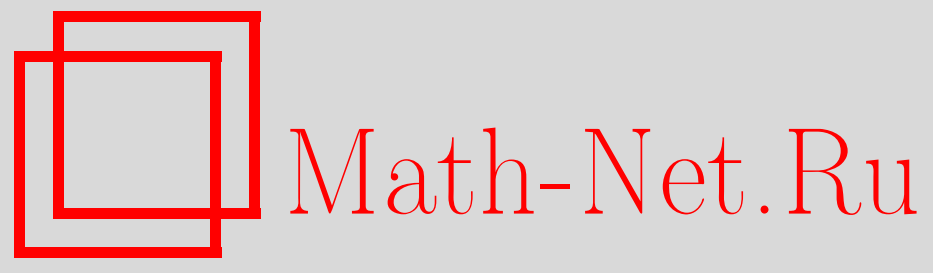

В. В. Стружанов, К. В. Бердников, Об устойчивости процесса полярно-симметричного деформирования тел из разупрочняющихся материалов, Вестн. Сам. гос. техн. ун-та. Сер. Физ.-мат. науки, 2014, выпуск 4(), 111-120

DOI: https://doi.org/10.14498/vsgtu1317

Использование Общероссийского математического портала MathNet.Ru подразумевает, что вы прочитали и согласны с пользовательским соглашением

http://www.mathnet.ru/rus/agreement

Параметры загрузки:

IP : 54.174 .149 .18

26 апреля 2023 г., 15:29:48

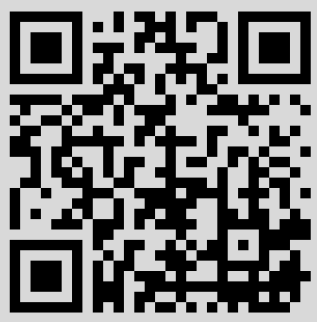


УДК 539.3

\title{
ОБ УСТОЙЧИВОСТИ ПРОЦЕССА ПОЛЯРНО- СИММЕТРИЧНОГО ДЕФОРМИРОВАНИЯ ТЕЛ ИЗ РАЗУПРОЧНЯЮЩИХСЯ МАТЕРИАЛОВ
}

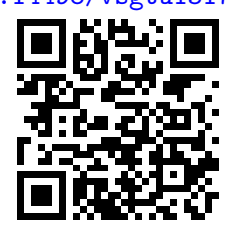

\section{В. В. Стружанов, К. В. Бердников}

Институт машиноведения УрО РАН,

Россия, 620049, Екатеринбург, ул. Комсомольская, 34.

\begin{abstract}
Аннотация
Рассматривается частный случай континуальных механических систем, деформируемых в условиях полярной симметрии напряжений и деформаций. Свойства материала описываются моделью Генки с разупрочнением при условии стесненного объемного деформирования (неположительности относительного изменения объема). Тогда единая кривая обладает падающей до нуля ветвью. Приведенные условия реализуются в задаче о расширении сферической полости в разупрочняющемся пространстве и при нагружении толстостенного сферического сосуда равномерным внешним давлением (батискаф, постепенно погружаемый на глубину). Исходя их формализма Лагранжа проведено исследование интегрального квадратичного функционала, представляющего собой приращение полной потенциальной энергии в виде лагранжиана в указанных задачах, что позволило сформулировать условия потери устойчивости квазистатически изменяющегося активного нагружения. Условие потери устойчивости имеет форму обращения в нуль второй вариации лагранжиана. Для рассмотренных задач получены множества возможных деформаций, возмущающих положение равновесия и не нарушающих кинематических связей, что обеспечило возможность выписать критерии потери устойчивости процесса деформирования в явном виде. Установлено, что потеря устойчивости процесса деформирования возможна только при достаточно развитой зоне разупрочненного материала.
\end{abstract}

Ключевые слова: упрочнение, разупрочнение, среда Генки, полярная симметрия, устойчивость, лагранжиан, матрица Гессе, вариации.

doi: http://dx.doi.org/10.14498/vsgtu1317

Введение. Учет разупрочнения, т. е. неустойчивых по Друккеру [1] состояний материала, как правило, приводит к тому, что при некотором значении внешней нагрузки процесс деформирования элемента конструкции или

(C) 2014 Самарский государственный технический университет.

\section{Образец для цитирования}

С тру жанов В. В., Бердни и в К. В. Об устойчивости процесса полярно-симметричного деформирования тел из разупрочняющихся материалов // Вестн. Сам. гос. техн. ун-та. Сер. Физ.-мат. науки, 2014. № 4 (37). С. 111-120. doi: 10.14498/vsgtu1317.

\section{Сведения об авторах}

Валерий Владимирович Стружанов (д.ф.-м.н., проф.; stru@imach.uran.ru), главный научный сотрудник, лаб. микромеханики материалов.

Кирилл Вячеславович Бердников (kir.berdnikov@mail.ru; автор, ведущий переписку), аспирант, лаб. микромеханики материалов. 
механической системы с элементом из разупрочняющегося материала теряет устойчивость $[2,3]$. Невозможность равновесия является признаком разрушения [4]. Таким образом, начало разрушения связывается с моментом потери устойчивости напряженно-деформированного состояния. В монографии [5] приведено исследование устойчивости квазистатического активного (без разгрузок) растяжения параллельно и последовательно соединенных стержней, часть из которых обладает эффектом деформационного разупрочнения. Анализ осуществлялся посредством применения аппарата математической теории катастроф [6, 7], справедливым для консервативных (градиентных) механических систем с конечным числом обобщенных координат. В этом случае он опирается на принцип стационарности потенциальной энергии, фактически вытекающий из уравнений Эйлера-Лагранжа при равенстве нулю кинетической энергии и независимости функции Лагранжа (лагранжиана) от скоростей и времени. Потеря устойчивости процесса растяжения наступает тогда, когда вторая вариация лагранжиана обращается в нуль, что происходит в момент вырождения матрицы Гессе (матрицы вторых производных лагранжиана по параметрам состояния системы - обобщенным координатам).

Исследование устойчивости процессов деформирования твердых тел, материал которых может работать и на стадии разупрочнения, осложняется тем, что деформируемые тела представляют собой механические системы с бесконечным числом переменных. В этом случае необходимо уже непосредственно использовать общий принцип гамильтоновой механики, а именно принцип наименьшего действия [8-10], на основе которого формулируются законы механики сплошных сред и других разделов механики и физики, излагающихся на языке теории поля [11]. В механике деформируемого твердого тела принципы гамильтоновой механики применимы, вообще говоря, для описания недиссипативных процессов. Однако известно [12], что при активном деформировании (без разгрузок) любой материал с формальной точки зрения можно рассматривать как нелинейно упругий. Поэтому в данном случае оправдано применение формализма Лагранжа и Гамильтона. При квазистатическом деформировании принцип наименьшего действия приводит к условию стационарности лагранжиана в положениях равновесия, а устойчивость равновесия определяется второй вариацией лагранжиана, который теперь представляет уже плотность потенциальной энергии.

В данной работе исследуется устойчивость полярно-симметричного процесса деформирования на примере задач о расширении сферической полости в пространстве и гидростатического давления на толстостенный сферический сосуд. Свойства материала описываются единой кривой с падающей ветвью (материал Генки с разупрочнением [13]). Сформулирован критерий потери устойчивости процесса деформирования при постепенно (квазистатически) возрастающих нагрузках, который можно использовать для прогнозирования разрушения рассматриваемых континуальных механических систем.

1. Лагранжиан при квазистатическом изотермическом процессе деформирования под действием внешней нагрузки (объемные силы отсутствуют). Лагранжиан представляет собой сумму двух работ

$$
W=W^{i}+W^{a}
$$

где $W^{i}$ - работа внутренних сил (энергия деформаций), $W^{a}$ - работа внеш- 
них сил, взятая со знаком минус (потенциальная энергия поверхностных сил). Будем считать, что материал, из которого изготовлено тело, является материалом Генки. Поэтому работа внутренних сил в элементарном объеме отождествляется со свободной энергией [14]. Тогда

$$
W^{i}=\int_{V} F d V
$$

где $F$ - свободная энергия, $V$ - объем тела.

При полярно-симметричном напряженно-деформированном состоянии имеют место только радиальные перемещения $u=u(r)$, а также определенные в сферической системе координат деформации $\varepsilon_{r}, \varepsilon_{\theta}=\varepsilon_{\varphi}$ и напряжения $\sigma_{r}$, $\sigma_{\theta}=\sigma_{\varphi}$. Все они зависят от одной переменной $r$ - расстояния от материальной точки до начала системы координат [15]. Функция свободной энергии для материала Генки имеет вид [14]

$$
F(\theta, \Gamma)=F\left(\varepsilon_{r}, \varepsilon_{\theta}, \varepsilon_{\varphi}\right)=\frac{1}{6} K \theta^{2}+\int_{0}^{\Gamma} G^{s}(\Gamma) \Gamma d \Gamma
$$

где $K$ - объемный модуль упругости, $\theta$ - относительное изменение объема материального элемента, $\Gamma$ - интенсивность деформаций сдвига [14], $G^{s}-$ секущий модуль единой кривой $T(\Gamma)=G^{s}(\Gamma) \Gamma[14]$ ( $T$ - интенсивность касательных напряжений). Для полярно-симметричного напряженно-деформированного состояния находим

$$
\theta=\varepsilon_{r}+2 \varepsilon_{\varphi}, \quad \Gamma=2\left(\varepsilon_{r}-\varepsilon_{\varphi}\right) / \sqrt{3}, \quad T=\left(\sigma_{r}-\sigma_{\varphi}\right) / \sqrt{3}
$$

Отметим, что выражение (1) при условии неположительности объемной деформации справедливо и на стадии разупрочнения (неустойчивого деформирования материала), когда единая кривая имеет ниспадающий до нуля участок [13].

Полярно-симметричное деформирование возможно тогда, когда на сферических поверхностях $S$, ограничивающих объем $V$, заданы либо равномерно распределенные силы $p$, действующие в радиальном направлении, либо одинаковые радиальные перемещения $v$ точкам границы. Потенциальная энергия внешних сил тогда составит величину

$$
W^{a}=-\int_{S_{\sigma}} p u d S=-p u_{s} S_{\sigma} .
$$

Здесь $S_{\sigma}$ - площадь сферической поверхности, на которой заданы силы, $u_{s}-$ перемещение точек границы $S_{\sigma}$, возникающие под действием сил.

Проверим корректность построенного лагранжиана. Найдем его первую вариацию. Имеем

$$
\begin{aligned}
\delta W=\delta W^{a}+\delta W^{i}=-p \delta u_{s} S_{\sigma}+\int_{V} & \left(\frac{\partial F}{\partial \varepsilon_{r}} \delta \varepsilon_{r}+2 \frac{\partial F}{\partial \varepsilon_{\theta}} \delta \varepsilon_{\theta}\right) d V= \\
& =-p \delta u_{s} S_{\sigma}+\int_{V}\left(\sigma_{r} \frac{d}{d r} \delta u+2 \sigma_{\theta} \frac{\delta u}{r}\right) d V .
\end{aligned}
$$


Здесь использованы соотношения Коши для полярно-симметричной деформации: $\varepsilon_{r}=d u / d r, \varepsilon_{\theta}=u / r=\varepsilon_{\varphi}$. Далее в сферической системе координат

$$
\begin{aligned}
\delta W^{i}=4 \pi \int_{R} r^{2} \sigma_{r} \frac{d}{d r} & \delta u d r+8 \pi \int_{R} r \sigma_{\theta} \delta u d r= \\
& =4 \pi\left(\left(r^{2} \sigma_{r} \delta u\right)_{R}-\int_{R} \frac{d}{d r}\left(r^{2} \sigma_{r}\right) \delta u d r+2 \int_{R} r \sigma_{\theta} \delta u d r\right) .
\end{aligned}
$$

Здесь $R$ - область изменения переменной $r$. Приравнивая теперь первую вариацию к нулю, находим уравнение равновесия

$$
2 r \sigma_{\theta}-\frac{d}{d r}\left(r^{2} \sigma_{r}\right)=0
$$

и граничное условие $\sigma_{r}=p$. Очевидно, что полученный результат согласуется с принципом Лагранжа $[15,16]$.

2. Критерий устойчивости. Рассмотрим некоторое положение равновесия деформируемого тела, возмутим его, задав вариации $\delta \varepsilon_{r}, \delta \varepsilon_{\varphi}, \delta \varepsilon_{\theta}$, и запишем приращения лагранжиана. Имеем [16]

$$
\Delta W=\delta W+\delta^{2} W+\cdots
$$

Поскольку исходная конфигурация находится в равновесии, $\delta W=0$. Следовательно знак приращения определяется знаком второй вариации.

Пусть для всех возможных (виртуальных) деформаций $\delta^{2} W>0$. Тогда $\Delta W>0$ при любых возмущениях положения равновесия. Функция $W$ имеет минимум и строго выпукла вниз. Равновесие устойчиво. Если всегда выполняется неравенство $\delta^{2} W<0$, то $\Delta W<0$. Функция $W$ имеет максимум и строго выпукла вверх. Равновесие абсолютно неустойчиво. Когда вторая вариация знаконеопределена, т. е. для одних возможных деформаций $\delta^{2} W>0$, а для других $\delta^{2} W<0$, лагранжиан $W$ имеет в положении равновесия седловую точку. При возмущениях в одних направлениях деформирование устойчиво, в других - неустойчиво. Таким образом, исходное равновесие неустойчиво, хотя и неабсолютно. Наконец, рассмотрим вариант положительной полуопределенности второй вариации, когда для некоторого набора возможных деформаций $\delta^{2} W=0$, а для всех остальных $\delta^{2} W>0$. В этом случае рассматриваемое состояние является пограничным, т. е. имеет место переход функции $W$ от выпуклости вниз к седловой точке (переход к неустойчивости). Следовательно, равенство $\delta^{2} W=0$ можно считать критерием, при выполнении которого процесс деформирования тела теряет устойчивость впервые при монотонном нагружении.

В рассматриваемых задачах полярно-симметричного деформирования

$$
\delta^{2} W=\delta^{2} W^{a}+\delta^{2} W^{i}=-p \delta^{2} u_{s} S_{\sigma}+\delta^{2} W^{i}
$$

где

$$
\delta^{2} W^{i}=\int_{V} \frac{\partial^{2} F}{\partial \varepsilon_{i} \partial \varepsilon_{j}} \delta \varepsilon_{i} \delta \varepsilon_{j} d V
$$


$\partial^{2} F / \partial \varepsilon_{i} \partial \varepsilon_{j}=H[F(r)]$ - матрица Гессе функции свободной энергии, компоненты которой определены для заданного положения равновесия. Отметим, что здесь индексы $i, j$ принимают значения $r, \theta, \varphi$. Матрица Гессе функции $F$ совпадает с матрицей тангенциальной жесткости материала $C^{p}[13]$ и имеет следующий вид:

$$
C^{p}=\left(\begin{array}{ccc}
C_{r r}^{p} & C_{r \theta}^{p} & C_{r \varphi}^{p} \\
C_{\theta r}^{p} & C_{\theta \theta}^{p} & C_{\theta \varphi}^{p} \\
C_{\varphi r}^{p} & C_{\varphi \theta}^{p} & C_{\varphi \varphi}^{p}
\end{array}\right),
$$

где

$$
\begin{gathered}
C_{r r}^{p}=\partial^{2} F / \partial \varepsilon_{r} \partial \varepsilon_{r}=\left(K+G^{p}\right) / 3, \\
C_{\theta \theta}^{p}=\partial^{2} F / \partial \varepsilon_{\theta} \partial \varepsilon_{\theta}=G^{s}+\left(K+G^{p}\right) / 3, \quad C_{\theta \theta}^{p}=C_{\varphi \varphi}^{p}, \\
C_{r \varphi}^{p}=C_{r \theta}^{p}=C_{\theta r}^{p}=C_{\varphi r}^{p}=\left(K-2 G^{p}\right) / 3 \\
C_{\theta \varphi}^{p}=C_{\varphi \theta}^{p}=-G^{s}+\left(K+G^{p}\right) / 3 .
\end{gathered}
$$

Здесь $G^{p}=G^{s}+\Gamma d G^{s} / d \Gamma$ - инкрементальный модуль, определяемый касательной к единой кривой $T \sim \Gamma$. Далее в случае полярно-симметричного деформирования тело должны ограничивать сферические поверхности $S_{\sigma}$ и $S_{u}$, где возможно задание либо равномерно распределенных радиальных сил, либо одинаковых радиальных перемещений. На поверхности $S_{u}$ имеем $p \delta u_{s}=0$ (вариация поверхностных перемещений равна нулю, так как $u_{s}$ фиксировано). На $S_{\sigma}$ имеем $\delta u_{s}=$ const и, следовательно, $\delta^{2} u_{s}=0$.

Таким образом, критерий потери устойчивости процесса деформирования (пограничное состояние) определяет равенство

$$
\int_{V} \delta \varepsilon^{\top} H(F) \delta \varepsilon d V=0
$$

где $\delta \varepsilon^{\top}=\left(\delta \varepsilon_{r}, \delta \varepsilon_{\theta}, \delta \varepsilon_{\varphi}\right)$ - вектор-строка, $\delta \varepsilon-$ соответствующий вектор-столбец.

Интеграл (2) можно представить суммой трех интегралов, вычисляемых соответственно в областях упругости, упрочнения (пластичности) и разупрочнения. Собственные числа матрицы $H(F)$ равны $K, 2 G^{p}, 2 G^{s}$. Так как в упругости и упрочнении $K>0, G^{p}>0, G^{s}>0$, матрица $H(F)$ является положительно определенной [17]. Тогда интегралы в областях упругости и упрочнения положительны. Поэтому при отсутствии зон разупрочнения процесс деформирования устойчив.

В области разупрочнения $G^{p}<0$, матрица $H(F)$ знаконеопределена и соответствующий интеграл может быть отрицательным. Следовательно, равенство нулю интеграла (2) и потеря устойчивости деформирования возможны только при достаточно развитой зоне разупрочнения материала.

Примечание. Вообще говоря, условие (2) является только необходимым. Для фиксирования факта потери устойчивости при монотонном нагружении следует исследовать на устойчивость ближайшее к исходному положение равновесия. Если оно неустойчиво, то условие (2) является и достаточным. В противном случае устойчивость деформирования сохраняется. 
3. Устойчивость процесса расширения сферической полости. Сферическая полость радиуса $а$ расположена в бесконечном упругопластическом пространстве, материал которого может работать на стадии разупрочнения. Пусть расширение полости осуществляется под действием равномерного внутреннего давления. Как было указано выше, потеря устойчивости процесса деформирования возможна при наличии зоны разупрочнения. Рассмотрим положение равновесия, когда зона разупрочнения ограничена сферическими поверхностями с радиусами $a$ и $r^{B}$, зона пластичности - поверхностями с радиусами $r^{B}$ и $r^{T}$, зона упругости - поверхностью радиусом $r^{T}$. Данное расположение зон вытекает из специфики напряженно-деформированного состояния [5]. Тогда критерий устойчивости (2) в сферической системе координат принимает вид

$$
\begin{aligned}
\int_{a}^{r^{B}} r^{2} \delta \varepsilon^{\top} \cdot H\left(F^{s t}\right) \cdot \delta \varepsilon d r+\int_{r^{B}}^{r^{T}} r^{2} \delta \varepsilon^{\top} & \cdot H\left(F^{h r}\right) \cdot \delta \varepsilon d r+ \\
& +\int_{r^{T}}^{\infty} r^{2} \delta \varepsilon^{\top} \cdot H\left(F^{e l}\right) \cdot \delta \varepsilon d r=0 .
\end{aligned}
$$

Здесь $H\left(F^{s t}\right), H\left(F^{h r}\right)$ и $H\left(F^{e l}\right)$ - матрицы инкрементальных модулей, компоненты которых являются функциями расстояния от начала координат и определены соответственно в областях разупрочнения, упрочнения и упругости в данном положении равновесия.

Для проверки равенства (3) необходимо иметь множество возможных деформаций, возмущающих положение равновесия и не нарушающих условий, при которых сформулирована задача. Такими ограничениями являются:

1) обращение в нуль деформаций на бесконечности;

2) неположительность объемной деформации;

3) удовлетворение виртуальными перемещениями уравнениям Ляме с нулевой правой частью (отсутствие объемных сил).

Таким условиям отвечают виртуальные перемещения типа $\alpha_{i} / r^{2}$, где $\alpha_{i}-$ произвольные малые числа. Отсюда возможные деформации, вычисляемые по соотношениям Коши, равны $\delta \varepsilon_{r}=-2 \alpha_{i} / r^{3}, \delta \varepsilon_{\theta}=\delta \varepsilon_{\varphi}=\alpha_{i} / r^{3}$. Подставляя эти значения в выражение (3), а также учитывая, что $H(F)=C^{p}$, после необходимых преобразований получаем равенство

$$
\int_{a}^{r^{B}} \frac{G_{s t}^{p}}{r^{4}} d r+\int_{r^{B}}^{r^{T}} \frac{G_{h r}^{p}}{r^{4}} d r+\frac{G}{3\left(r^{T}\right)^{3}}=0 .
$$

Здесь $G$ - модуль сдвига в упругости, $G_{h r}^{p}>0$ - касательная к единой кривой на стадии упрочнения, $G_{s t}^{p}<0$ - касательная на стадии разупрочнения.

4. Устойчивость процесса гидростатического сжатия сферического сосуда. Рассмотрим толстостенный сферический сосуд, находящийся под воздействием равномерного внешнего давления. Внутренний и внешний радиусы сферических поверхностей, ограничивающих сосуд, соответственно $a$ и $b$. С учетом распределения зон упругости, упрочнения и разупрочнения [5] критерий (2) принимает вид 


$$
\begin{aligned}
\int_{a}^{r^{B}} r^{2} \delta \varepsilon^{\top} \cdot H\left(F^{s t}\right) \cdot \delta \varepsilon d r+\int_{r^{B}}^{r^{T}} r^{2} \delta \varepsilon^{\top} \cdot H\left(F^{h r}\right) \cdot \delta \varepsilon d r+ & \\
& +\int_{r^{T}}^{b} r^{2} \delta \varepsilon^{\top} \cdot H\left(F^{e l}\right) \cdot \delta \varepsilon d r .
\end{aligned}
$$

Возможные перемещения, удовлетворяющие приведенным выше условиям, есть линейная комбинация $\alpha_{i} / r^{2}+\beta_{j} r\left(\alpha_{i}, \beta_{j}\right.$ - произвольные малые числа). После подстановки всех необходимых значений в выражение (4) и проведения преобразований с учетом независимости $\alpha_{i}$ и $\beta_{j}$ получаем два равенства:

$$
\begin{gathered}
\int_{a}^{r^{B}} \frac{G_{s t}^{p}}{r^{4}} d r+\int_{r^{B}}^{r^{T}} \frac{G_{h r}^{p}}{r^{4}} d r+\frac{G}{3}\left(\frac{1}{\left(r^{T}\right)^{3}}-\frac{1}{b^{3}}\right)=0 \\
\int_{a}^{r^{B}}\left(3 K-G_{s t}^{p}\right) r^{2} d r+\int_{r^{B}}^{r^{T}}\left(3 K-G_{h r}^{p}\right) r^{2} d r+\frac{1}{3}(3 K-G)\left(b^{3}-\left(r^{T}\right)^{3}\right)=0 .
\end{gathered}
$$

Выполнение хотя бы одного из этих равенств прогнозирует неустойчивость равновесия.

Заключение. С использованием лагранжева формализма исследована устойчивость процесса полярно-симметричного деформирования твердых тел при стесненных условиях объемного деформирования, свойства материала которых описываются моделью Генки с разупрочнением. В задачах о расширении сферической полости в пространстве и гидростатическом сжатии толстостенного сферического сосуда из разупрочняющегося материала определены множества возможных деформаций, возмущающих положение равновесия и не нарушающих кинематических связей, которые позволяют выписать критерии потери устойчивости процесса деформирования для указанных задач.

Благодарности. Работа выполнена при поддержке РФФИ (проект № 13-08-00186-а).

\section{ORCID}

Валерий Владимирович Стружанов: http://orcid.org/0000-0002-3669-2032

Кирилл Вячеславович Бердников: http://orcid.org/0000-0002-8505-6892

\section{БИБЛИОГРАФИЧЕСКИЙ СПИСОК}

1. Drucker D. C. A definition of a stable inelastic material // ASME J. Appl. Mech., 1959. vol. 26. pp. 101-195.

2. Стружанов В. В., Бурмашева Н. В. Вычислительная процедура нахождения предельных значений параметров нагружения механических систем // Вычислительная механика сплошных сред, 2011. Т. 4, № 4. С. 107-113. doi: 10.7242/1999-6691/2011.4.4.45.

3. Стружанов В. В., Просвиряков Е. Ю. Растяжение с кручением. Сообщение 2: Устойчивость процесса деформирования образца в механической системе. Жесткое и мягкое нагружения // Вестн. Сам. гос. техн. ун-та. Сер. Физ.-мат. науки, 2008. №2(17). C. 77-86. doi: $10.14498 / v s g t u 403$.

4. Седов Л. И. Механика сплошной средъ. Т. 1. М.: Наука, 1970. 492 с.

5. Стружанов В. В., Миронов В. И. Деформачионное разупрочнение материала в элеменmax конструкций. Екатеринбург: УрО РАН, 1995. 192 с.

6. Гилмор Р. Прикладная теория катастроф. В 2-х книгах. Кн. 1. М.: Мир, 1984. 350 с.

7. Постон Т., Стюарт И. Теория катастроф и её приложения. М.: Мир, 1980. 608 с. 
8. Арнольд В. И. Математические методы классической механики. М.: Наука, 1979. $432 \mathrm{c}$.

9. тер Хаар Д. Основъ гамильтоновой механики. М.: Наука, 1974. 224 с.

10. Парс Л. А. Аналитическал динамика. М.: Наука, 1971. 636 с.

11. Ковалев В. А., Радаев Ю. Н. Математические модели и современные физические теории поля // Изв. Сарат. ун-та. Нов. сер. Сер. Математика. Механика. Информатика, 2009. Т. 9, № 4(2). С. 41-94.

12. Ильюшин А. А. Пластичность. Часть 1. Упруго-пластические деформации. М., Л.: ОГИЗ, 1948. 378 с.

13. Стружанов В. В., Бердников К. В. Об определяющих соотношениях среды Генки для разупрочняющегося материала при диагональном тензоре деформаций // Вестн. Сам. гос. техн. ун-та. Сер. Физ.-мат. науки, 2012. №3(28). С. 72-80. doi: 10.14498/ vsgtu1115.

14. Лурье А. И. Теория упругости. М.: Наука, 1970. 940 с.

15. Хан Х. Теория упругости. Основы линейной теории и её применения. М.: Мир, 1988. $344 \mathrm{c}$.

16. Васидзу К. Вариационные методы в теории упругости и пластичности. М.: Мир, 1987. 542 c.

17. Хорн Р., Джонсон Ч. Матричный анализ. М: Мир, 1989. 655 с.

Поступила в редакцию $24 / \mathrm{IV} / 2014$;

в окончательном варианте - 11/VII/2014;

принята в печать $-27 / \mathrm{XI} / 2014$.

Vestn. Samar. Gos. Techn. Un-ta. Ser. Fiz.-mat. nauki

[J. Samara State Tech. Univ., Ser. Phys. \& Math. Sci.] 2014. Issue 4(37). Pp. 111-120

ISSN: 2310-7081 (online), 1991-8615 (print)

doi: http://dx.doi.org/10.14498/vsgtu1317

MSC: 74C10

\title{
ON A STABILITY OF POLAR SYMMETRICAL DEFORMATION OF BODIES FROM SOFTENING MATERIALS
}

\section{V. Struzhanov, K. V Berdnikov}

Institute of Engineering Science, Ural Branch of RAS,

34, Komsomolskaya st., Ekaterinburg, 620049, Russian Federation.

\begin{abstract}
Special case of continuum mechanical systems is considered. It is believed that deforming is carried out under conditions of polar symmetry of stresses
\end{abstract}

(C) 2014 Samara State Technical University.

\section{How to cite Reference}

Struzhanov V. V., Berdnikov K. V. On a stability of polar symmetrical deformation of bodies from softening materials, Vestn. Samar. Gos. Tekhn. Univ., Ser. Fiz.-Mat. Nauki [J. Samara State Tech. Univ., Ser. Phys. \& Math. Sci.], 2014, no. 4(37), pp. 111-120. doi: 10.14498/vsgtu1317. (In Russian)

\section{Authors Details}

Valery V. Struzhanov (Dr. Phys. \& Math. Sci.; stru@imach.uran.ru), Chief Researcher, Lab. of Matherial Micromechanics.

Kirill V. Berdnikov (kir.berdnikov@mail.ru; Corresponding Author), Postgraduate Student, Lab. of Matherial Micromechanics. 
and strains. Also it is assumed that material properties are described by Hencky model with softening under nonpositivity of volume deformation. Then union curve has region decreasing to zero. Aforementioned conditions are realized in such problems as expansion of spherical cavity in softening space and deforming of thick-walled spherical vessel by equable external pressure (it maybe bathyscaphe which is gradually submerged to the deep). Based on the Lagrange formalism integral quadratic functional is investigated. This functional is increment of total potential energy in the form of Lagrangian for mentioned problems. This study allows to formulate conditions of buckling for active loading which changes quasistatically. For considered problems sets of possible deformations are obtained. These possible deformations perturb the equilibrium position and do not break kinematic constraints. Obtained sets of possible deformations allow to write criterion of buckling of deformation process in explicit form for mentioned problems. It is established that only with sufficiently developed softening zone buckling of deformation process is possible.

Keywords: hardening, softening, Hencky environment, polar symmetry, stability, Lagrangian, Hesse matrix, variations.

doi: http://dx.doi.org/10.14498/vsgtu1317

Acknowledgments. This work has been supported by the Russian Foundation for Basic Research (project no. 13-08-00186-a).

\section{ORCID}

Valery V. Struzhanov: http://orcid.org/0000-0002-3669-2032

Kirill V. Berdnikov: http://orcid.org/0000-0002-8505-6892

\section{REFERENCES}

1. Drucker D. C. A definition of a stable inelastic material, ASME J. Appl. Mech., 1959, vol. 26, pp. 101-195.

2. Struzhanov V. V., Burmasheva N. V. Computational procedure for determining the limiting values of the loading parameters of mechanical systems, Computational Continuum Mechanics, 2011, vol. 4, no. 4, pp. 107-113 (In Russian). doi: 10.7242/1999-6691/2011.4. 4.45 .

3. Struzhanov V. V., Prosviryakov E. Yu. Tension with torsion. Part 2. Deformation process stability of a sample in a mechanical system. Rigid and soft loadings, Vestn. Samar. Gos. Tekhn. Univ. Ser. Fiz.-Mat. Nauk, 2008, no. 2(17), pp. 77-86 (In Russian). doi: 10.14498/ vsgtu403.

4. Sedov L. I. Mekhanika sploshnoi sredy [Mechanics of the continuum media], vol. 1. Moscow, Nauka, 1970, 492 pp. (In Russian)

5. Struzhanov V. V., Mironov V. I. Deformatsionnoe razuprochnenie materiala $v$ elementakh konstruktsii [Deformational Softening of Material in Structural Elements]. Ekaterinburg, Ural Branch of RAS, 1995, 192 pp. (In Russian)

6. Gilmore R. Catastrophe theory for scientists and engineers, A Wiley-Interscience Publication. New York, John Wiley \& Sons, 1981, xvii+666 pp.

7. Poston T., Stewart I. Catastrophe theory and its applications, Surveys and Reference Works in Mathematics, vol. 2. London, San Francisco, Melbourne, Pitman, 1978, xviii+491 pp.

8. Arnol'd V. I. Mathematical Methods of Classical Mechanics, Graduate Texts in Mathematics, vol. 60. New York, Springer Science \& Business Media, 1989, xvi+520 pp.. doi: 10.1007/ 978-1-4757-1693-1.

9. ter Haar D. Elements of Hamiltonian Mechanics. Amsterdam, North-Holland Publishing Co., 1961, viii+191 pp.

10. Pars L. A. A treatise on analytical dynamics. London, Heinemann Educational Books Ltd., 1965, xxi+641 pp. 
11. Kovalev V. A., Radayev Yu. N. Mathematical models and contemporary theories of physical fields, Izv. Saratov. Univ. Mat. Mekh. Inform., 2009, vol. 9, no. 4(2), pp. 41-94 (In Russian).

12. Il'iushin A. A. Plastichnost'. Chast' 1. Uprugo-plasticheskie deformatsii [Plasticity. Part 1: Elastic-plastic deformations]. Moscow, Leningrad, OGIZ, 1948, 378 pp. (In Russian)

13. Struzhanov V. V., Berdnikov K. V. On defining relations for the Hencky environment of softening of the material under diagonal stress tensor, Vestn. Samar. Gos. Tekhn. Univ. Ser. Fiz.-Mat. Nauki, 2012, no. 3(28), pp. 72-80 (In Russian). doi: 10.14498/vsgtu1115.

14. Lurie A. I. Theory of Elasticity. Berlin, Heidelberg, New York, Springer, 2005, 1050 pp.. doi: 10.1007/978-3-540-26455-2.

15. Khan Kh. Teoriya uprugosti. Osnovy lineinoy teorii $i$ ee primeneniya [The theory of elasticity. Fundamentals of linear theory and its applications]. Moscow, Mir, 1988, 344 pp. (In Russian)

16. Washizu K. Variational methods in elasticity and plasticity, International Series of Monographs in Aeronautics and Astronautics, vol.9. Oxford, Pergamon Press, 1968, $\mathrm{x}+349 \mathrm{pp}$.

17. Horn R. A., Johnson C. R. Matrix analysis. Cambridge, Cambridge University Press, 1985, xiii+561 pp.

Received 24/IV/2014;

received in revised form 11/VII/2014;

accepted 27/XI/2014. 